\title{
Comparison of laboratory and in-flight performance of infared array camera (IRAC) detector arrays on Spitzer Space Telescope
}

Judith L. Pipher, Craig W. McMurtry, William J. Forrest, Craig R. McCreight, Mark E. McKelvey, et al.

Judith L. Pipher, Craig W. McMurtry, William J. Forrest, Craig R. McCreight, Mark E. McKelvey, Robert E. McMurray, Roy R. Johnson, Giovanni G. Fazio, Joseph L. Hora, Lori E. Allen, Matthew L. N. Ashby, Pauline Barmby, Lynne K. Deutsch, Jiasheng Huang, Massimo Marengo, S. Thomas Megeath, Michael A. Pahre, Brian Michael Patten, Zhong Wang, Steven P. Willner, William F. Hoffmann, Samuel Harvey Moseley, Richard G. Arendt, Danny J. Krebs, Peter R. Eisenhardt, Daniel Stern, Varoujan Gorjian, Bidushi Bhattacharya, William J. Glaccum, Mark David Lacy, Patrick J. Lowrance, Sean J. Carey, Seppo Jalmari Laine, John R. Stauffer, Jason A. Surace, William T. Reach, Gillian Wilson, "Comparison of laboratory and in-flight performance of infared array camera (IRAC) detector arrays on Spitzer Space Telescope," Proc. SPIE 5487, Optical, Infrared, and Millimeter Space Telescopes, (12 October 2004); doi: 10.1117/12.551599

Event: SPIE Astronomical Telescopes + Instrumentation, 2004, Glasgow, United Kingdom 


\title{
Comparison of laboratory and in-flight performance of InfraRed Array Camera (IRAC) detector arrays on Spitzer Space Telescope
}

\author{
Judith L. Pipher*,a, Craig W. McMurtry ${ }^{\mathrm{a}}$, William J. Forrest ${ }^{\mathrm{a}}$, Craig R. McCreight ${ }^{\mathrm{b}}$, Mark E. \\ McKelvey $^{\mathrm{b}}$, Robert E. McMurray Jr. ${ }^{\mathrm{b}}$, Roy R. Johnson ${ }^{\mathrm{b}}$, Giovanni G. Fazio ${ }^{\mathrm{c}}$, Joseph L. Hora, Lori \\ E. Allen ${ }^{c}$, Matthew L. N. Ashby ${ }^{c}$, Pauline Barmby ${ }^{c}$, Lynne K. Deutsch ${ }^{\text {,d }}$, Jiasheng Huang ${ }^{c}$, \\ Massimo Marengo ${ }^{c}$, S. Thomas Megeath ${ }^{c}$, Michael A. Pahre ${ }^{c}$, Brian M. Patten ${ }^{c}$, Zhong Wang, \\ Steven P. Willner ${ }^{\mathrm{c}}$, William F. Hoffmann ${ }^{\mathrm{e}}$, S. Harvey Moseley ${ }^{\mathrm{f}}$, Richard G. Arendt ${ }^{\mathrm{f}}$, Danny J. \\ Krebs $^{\mathrm{f}}$, Peter Eisenhardt ${ }^{\mathrm{g}}$, Daniel Stern ${ }^{\mathrm{g}}$, Varoujan Gorjian ${ }^{\mathrm{g}}$, Bidushi Bhattacharya ${ }^{\mathrm{h}}$, William J. \\ Glaccum $^{\mathrm{h}}$, Mark Lacy ${ }^{\mathrm{h}}$, Patrick J. Lowrance ${ }^{\mathrm{h}}$, Sean J. Carey ${ }^{\mathrm{h}}$, Seppo Laine ${ }^{\mathrm{h}}$, John Stauffer ${ }^{\mathrm{h}}$, Jason \\ A. Surace ${ }^{\mathrm{h}}$, William T. Reach ${ }^{\mathrm{h}}$, Gillian Wilson ${ }^{\mathrm{h}}$ \\ ${ }^{a}$ University of Rochester, Rochester, NY 14627 USA \\ bNASA Ames Research Center, Moffett Field, CA 94035 USA \\ ${ }^{\mathrm{c}}$ Smithsonian Astrophysical Observatory, Cambridge MA 02138 \\ deceased \\ eUniversity of Arizona, Tucson AZ 85721 USA \\ ${ }^{\mathrm{f}}$ Goddard Space Flight Center, Greenbelt, MD, 20771 USA \\ gJPL, Pasadena CA, 91109 USA \\ ${ }^{\mathrm{h}}$ Spitzer Science Center, Pasadena, CA 91125 USA
}

\begin{abstract}
The Infrared Array Camera (IRAC) on Spitzer Space Telescope includes four Raytheon Vision Systems focal plane arrays, two with InSb detectors, and two with Si:As detectors. A brief comparison of pre- flight laboratory results vs. inflight performance is given, including quantum efficiency and noise, as well as a discussion of irregular effects, such as residual image performance, "first frame effect", "banding", "column pull-down" and multiplexer bleed. Anomalies not encountered in pre-flight testing, as well as post-flight laboratory tests on these anomalies at the University of Rochester and at NASA Ames using sister parts to the flight arrays, are emphasized.
\end{abstract}

Keywords: Spitzer Space Telescope, Mid-wave infrared (MWIR), Long-wave infrared (LWIR), Detector Array, InSb, Si:As IBC.

\section{INTRODUCTION}

The Spitzer Space Telescope was successfully launched on August 25, 2003. IRAC, the InfraRed Array Camera, is a four-channel camera on Spitzer which obtains simultaneous 5.2' square images at wavelengths of $3.6 \mu \mathrm{m}$ (Channel 1), $4.5 \mu \mathrm{m}$ (Channel 2), $5.8 \mu \mathrm{m}$ (Channel 3), and $8.0 \mu \mathrm{m}$ (Channel 4). There are a number of papers describing the instrument, as well as the pre-flight and in-flight performance of IRAC detector arrays already in the literature $1,2,3,4,5,6,7$ and in this volume ${ }^{8}$. Therefore we will only summarize or mention characterization that has been discussed in detail elsewhere, and concentrate our efforts here on the attempts made to understand some of the more anomalous behaviors affecting these arrays. Post-flight tests were conducted not only during IOC (In-Orbit Checkout) as calibrations of the IRAC instrument were obtained, but also at laboratories at University of Rochester (UR) and NASA Ames on sister parts to the flight arrays.

*jlpipher@astro.pas.rochester.edu Phone 1-585-275-4402. 


\section{PERFORMANCE OF IRAC ARRAYS}

Fazio et al. $^{7}$ summarize the IRAC point source sensitivity for the four IRAC arrays under low background conditions, and for every frame time used on Spitzer. More detailed information can also be found in Hora et al. ${ }^{8}$ and in the Spitzer Observer's Manual at http://ssc.spitzer.caltech.edu/documents/som/ as well as in the webpage references given in that document. The IRAC detector arrays, developed for IRAC by Raytheon Vision Systems ${ }^{9}$, employ InSb detectors (Channel 1, 3.6 $\mu \mathrm{m}$ and Channel 2, $4.5 \mu \mathrm{m}$ ) and Si:As IBC detectors (Channels 3, $5.8 \mu \mathrm{m}$ and Channel 4, 8.0 $\mu \mathrm{m}$ ). All of the arrays exhibit relatively high quantum efficiencies ( $\geq 86 \%$ for the InSb arrays, $45 \%$ for Channel 3 , and $70 \%$ for Channel 4), and the measured read noise is $8.1,6.8,13.0 \mathrm{e}^{-}$for $200 \mathrm{~s}$ frame time measurements in Channels 1,2 , and 3 respectively, and $6.6 \mathrm{e}$ - for $50 \mathrm{~s}$ frame time measurements in Channel 4 . Ratios of the measured point source detection threshold to the pre-flight determination are $0.69,0.75,1.60$, and 1.31 in Channels $1,2,3$, and 4, respectively, for 30s low background frames. Channels 1 and 2 perform as expected, and in fact are slightly better in sensitivity than the advertised pre-flight sensitivity values. However, Channels 3 and 4 are somewhat less sensitive, and a major focus of the post-flight laboratory characterization has been directed toward understanding this and related results. Post-flight laboratory measurements have also addressed anomalies with the IRAC arrays discovered in flight.

\section{ANOMALIES OF THE FLIGHT ARRAYS}

Despite the almost flawless performance of IRAC, and the incredible sensitivity of the four cameras, we found a few anomalies that were not known pre-flight, as well as those that have already been described ${ }^{3,4}$ and are detailed in the Spitzer Observer's Manual. Anomalies include the phenomena known as "long-lived latent images" in Channels 1 and 4; "banding" especially in Channels 3 and 4; column "pull-down"; "lost light" or throughput anomaly in Channel 3 and to some extent Channel 4; as well as multiplexer bleed; first frame effect, and residual (latent images) in all channels. Several of these prove to be related effects.

\subsection{First Frame Effect}

As described in references 4 and 5, the first several dark image frames from IRAC arrays have a higher median level than subsequent frames. The magnitude of the so-called "first frame effect" depends on the array's state during the interval preceding the image, including the time elapsed since a prior frame, the particular frame time and Fowler number (see Spitzer Observer's Manual) chosen. Whether a given pixel is being reset, integrating, or read out, there are voltage changes across the detector node, and a hysteresis-like behavior arises as the charges redistribute in the multiplexer. This effect is not uniform over the array and is documented in the Spitzer User's Manual. It can be calibrated, in principle: nonetheless, the first image in an AOR may not have a suitable dark correction ${ }^{10}$. Pre-flight calibrations of the "first-frame effect" have been improved by IOC calibrations. As we discuss in $\$ 3.2 .1$, the first frame effect for Channel 1 is not as simply explained as for the other IRAC channels, and it can influence the Channel 1 "long-lived latent" images discovered during IOC.

\subsection{Long-lived Latent Images}

\subsubsection{InSb Channel 1 Array}

The IRAC instrument was assembled and tested at the Goddard Space Flight Center (GSFC) and a battery of tests were conducted from November 1999 to August 2000. Following analysis of the tests, we realized there was anomalous, hysteresis-like behavior in the Channel 1 array, which was an extreme example of what became known as the "first frame effect" seen in all arrays. Tests at UR showed conclusively that the anomalous behavior, namely an extremely large first frame level, and decay to a residual level rather than $0 \mathrm{DN}$ as expected for these low dark current devices, could be reproduced if there was an open connection in the clamp circuit (the purpose of which was to keep a detector array from moving rapidly into forward bias when no row is selected). It was determined that by appropriate clocking of the array (minimizing the amount of time no row is selected), setting both the clamp clock levels to $0 \mathrm{~V}$, and increasing the bias across the diode to negate any tendency toward forward bias, that the extreme first frame effect could be minimized. Rather than delaying the schedule, this work-around was adopted, and the IRAC cryostat was not opened up to correct the open connection: in fact, once the IRAC experiment was cooled in December 2000 at Ball, it 
remained cold until launch throughout the Ball and Lockheed integration tests. Even when the Channel 1 readout operation was optimized as mentioned above, it showed a more extreme first frame effect than the other channels.

During IOC, it was found that the Channel 1 array exhibited extremely long-lived "latent images", lasting more than 5 hours, and even days. Normal latent images (discussed in $\S 3.4$ below and in detail by Hora et al. ${ }^{5}$ ), due to charge trapping in the InSb material, decay away relatively quickly. As discussed here, we believe the long lived "latent images" in Channel 1 to be due to a small change in the stray gate-source capacitance of the output FET of the multiplexer unit cells. Thus in this case the term "latent image" is a misnomer.

Even though the Channel 2 array was of similar detector-type and heritage to that of Channel 1, this array did not exhibit similar long-lived latent images. Tests at UR concentrated on the obvious difference between the Channel 1 and 2 arrays, namely a working clamp circuit in Channel 2, but not in Channel 1. A detector array with an ROIC (read-out integrated circuit) from the same multiplexer lot run as that of the Channel 1 array, but with different detector material, was tested with the clamp clocks turned both off and on (the array tested at UR had a working clamp circuit). This device was the only one for which we were able to reproduce "long-lived latent images". Long-lived latent images were generated when either the Vggcl clock or the Vddcl bias was turned off, so that when no row is selected there wasn't a clamp to prevent the array from going into forward bias. Long-lived latent images were not generated with the clamp clock on. Demonstration of these two situations in the laboratory is shown in Figure 1.
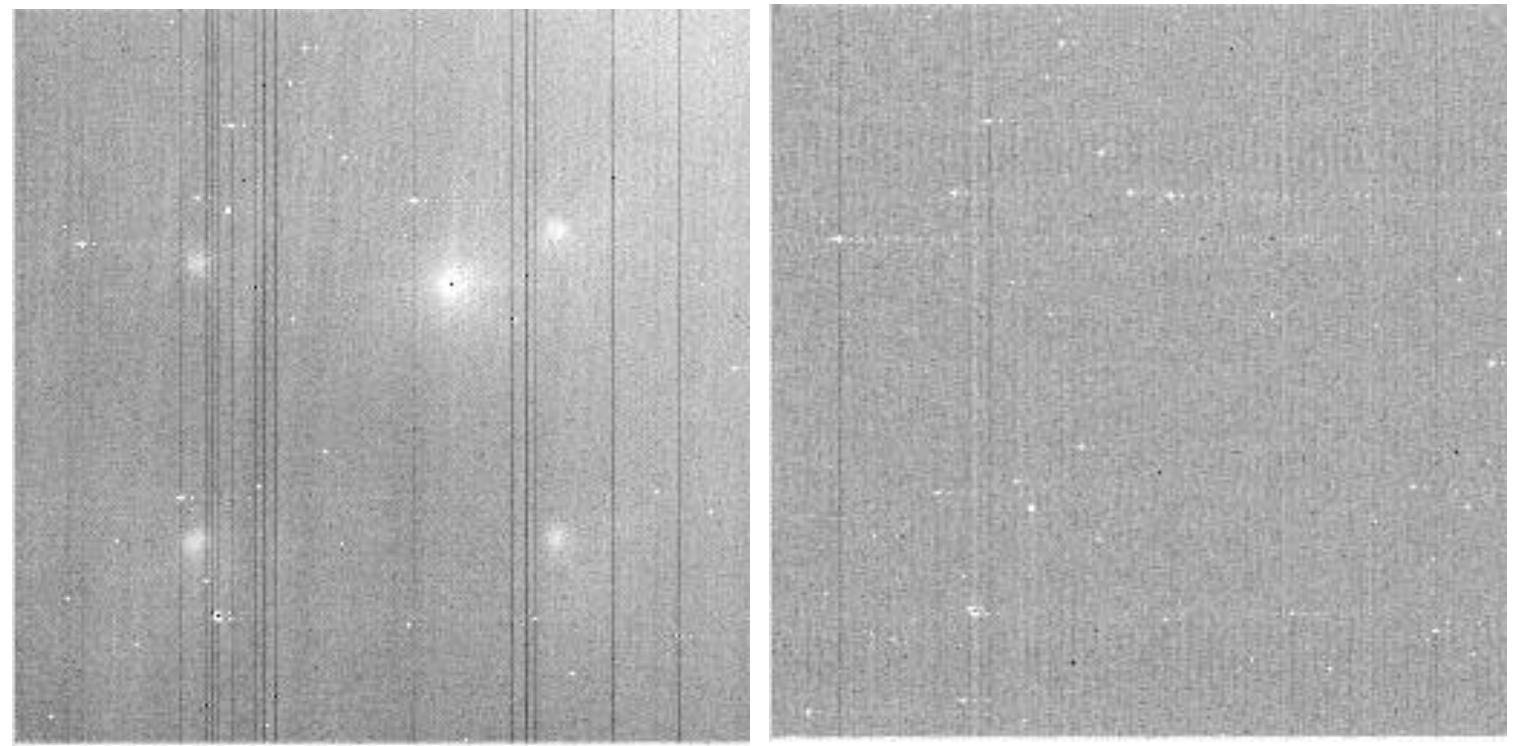

Figure 1. Images from UR laboratory tests on InSb array with sister ROIC to Channel 1. Left: image with long lived latents 30 minutes following exposure, Vddcl off. The four point source images in a square are the areas where there was prior bright exposure. (A bright PED mars the image). Right: image taken under same conditions, but Vddcl on.

There was no dependence on the wavelength of source illumination. The long lived latent images appear to originate in the multiplexer and not in the InSb detector material. Gain changes in the source follower output FET of the unit cells were not observed. Rather, bright source illumination causes the affected detector pixels to go into forward bias when the clamp is off, and no row is selected. For a pixel in forward bias, current flows in a pixel and the unit cell of the ROIC. This current allows charge trapping to occur at the gate of the multiplexer source follower output FET, which causes a gate-to-source capacitance change at the unit cell output FETs. There is no depopulation mechanism for the trapped charge under normal operation: hence when brief bright point source illumination of the Channel 1 array occurs, the overall pixel capacitance (which is comprised of a detector, bump bond, and multiplexer component) is semi-permanently decreased. Subsequent exposure to a uniform low background, the array, with the prior point source region now at lower capacitance, appears to have an apparent "latent image" at that position. The excessive first frame effect in Channel 1 can also add to this apparent latent. After producing long lived "latent images" in the laboratory 
device, then turning the clamp back on, mostly eliminates the apparent latent images, but turning the clamp off again without further exposure to light, brings the "latents" back. Similar experiments were conducted on a device with InSb from the same wafer as for the Channel 1 array, but the detector was bump-bonded to an ROIC from a different lot. Long-lived latent images were not produced in any instance. Both experiments indicate that the source of the Channel 1 long-lived "latents" is the multiplexer, and not the detector material as for normal latent images.

\subsubsection{Si:As Channel 4 Array}

Channel 4 arrays also exhibit long-lived residual images (they last many days without decline even through power cycles). While the magnitude of the effect is small, they can seriously impact observational strategy. The latent images $\left(10^{\text {th }}-11^{\text {th }}\right.$ magnitude, for a $\mathrm{K}=4^{\text {th }}$ magnitude source and brighter) are almost hidden in the noise and background variations in the array. To analyze quantitatively the strength of the latent images, M. Marengo found it necessary to correct for the background variations. In Figure 2 below, a latent-free dark was subtracted from one with latent images, leading to the result in the bottom right panel. See also Hora et al. ${ }^{8}$

Figure 2. Saturated sources in Channel 4 are shown in upper left panel (central part of saturated source appears dark). Background variations removed from source frame on upper right, faint latent images on lower right.

To date neither the UR nor Ames labs have been able to replicate this effect in the laboratory. A summary of the measurements to date is provided below.

After the launch of Spitzer, Ames conducted tests on three parts, to try to reproduce effects seen in flight. The first device tested had a multiplexer made from a die which was adjacent to the that of the Channel 4 multiplexer but with a detector from another lot run. Next was tested a device with a detector substrate from the same lot as the flight part. Finally, a device with
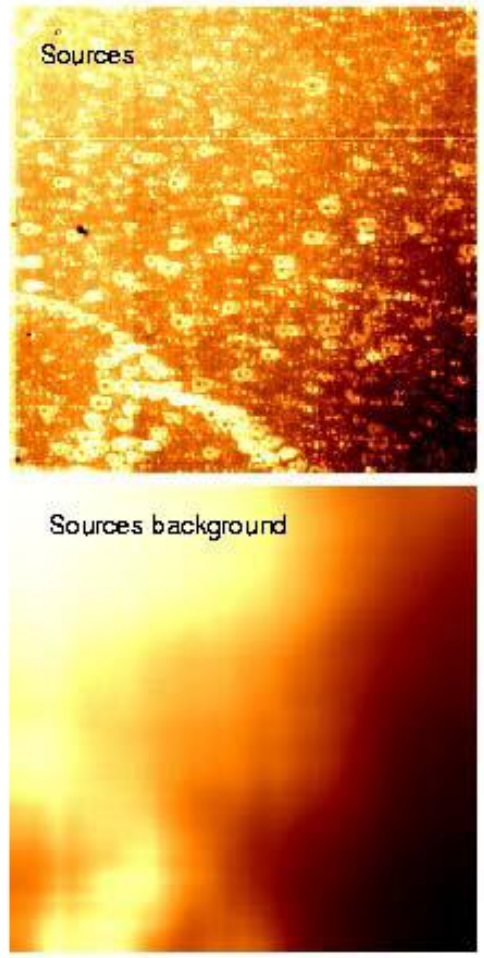
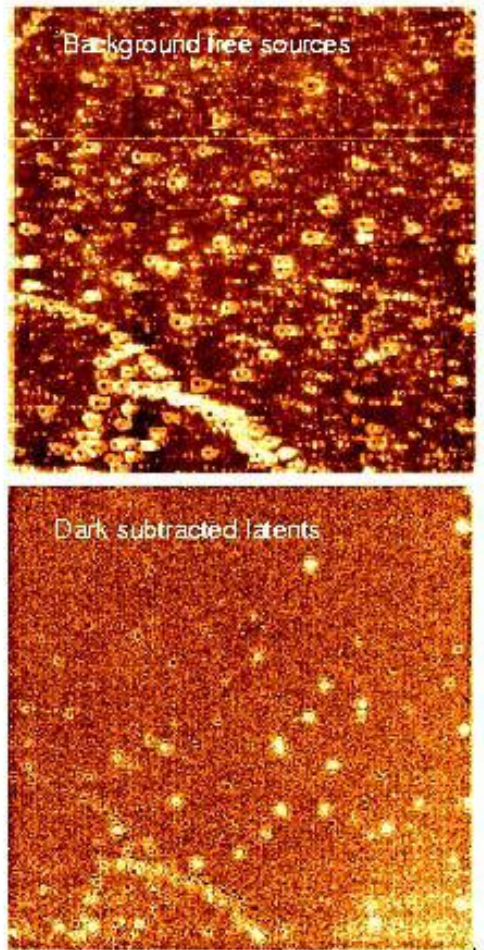

neither multiplexer nor detector from the same runs as the Channel 4 array was tested. The flight conditions were emulated (i.e. a point source illumination through the flight $f /$ number in the presence of background irradiance, approximately that of the zodiacal background). Independently controlled spot and background sources were used. Test arrays were heavily over-saturated for extended periods, without effect. Two arrays were then shipped to UR for test.

UR tests concentrated on multiplexer behaviors as the cause, following their experiences with the Channel 1 array. First the Ames tests were repeated, utilizing the exact clock timing that Spitzer employs, to no avail. Then, in a similar set of experiments as for Channel 1, various bias and/or clock levels were varied, but there were no long-lived latent images produced. Present experiments are concentrating on changes in the reset level that could have been caused by a cosmic ray upset. That experiment is still in progress. 


\subsubsection{Long Lived Latent Amelioration in Flight}

During IOC, ameliorations and pre-emptive actions for anomalies were developed ${ }^{10}$.

The Channel 1 long-lived latents were discovered following a high gain antenna downlink, where the array was staring at the same place in the sky at a Ks magnitude $=6.4$ star for the better part of an hour. The array is reset every $0.2 \mathrm{~s}$ when it stares at one place while not in data acquisition mode. Originally, we had planned to employ the cold shutter to block off all light during such periods. However, it was decided by the project not to activate a cold shutter for IRAC during flight to reduce risk ${ }^{8}$. After several tests to determine the best strategy, including changing to subarray mode and resetting every $0.01 \mathrm{~s}$ (which did not help), we found that anneals helped eliminate long-lived latents. At the beginning of each campaign all channels are annealed, and Channels 1 and 4 are annealed following every downlink.

A method not involving anneals was developed at UR. Changing biases Vdet and Vrstuc, hence the applied bias across the diodes, from $0 \mathrm{~V}$ to $1 \mathrm{~V}$ to nominal $0.75 \mathrm{~V}$, at the same time the reset voltage Vrstuc is changed from -3.0

to -3.5 , to $-3.5 \mathrm{~V}$, with 10 resets each time, leads to a reduction in the long-lived latents to $10-30 \%$ of their previous levels. However, the UR method was not implemented for Spitzer since the current anneal method provides a full removal of long-lived latent images.

The Spitzer Observer's Manual outlines observing strategies designed to mitigate production of these long-lived latent image effects.

\subsection{Sensitivity (Flux Throughput Anomaly) and Banding in Channels 3 and 4}

Sensitivity to point and extended sources is not equal to pre-launch ${ }^{5}$ predictions. As noted in $\S 2$, the ratio of the point source detection threshold to the pre-launch expectation, based on laboratory tests at UR, Ames, and GSFC are 0.69, $0.75,1.60$, and 1.31 in Channels 1 through 4 respectively, for low background $30 \mathrm{sec}$ frames. This decrease in sensitivity in Channels 3 and 4 is referred to as the "flux throughput" anomaly. As pointed out by Hora et al. ${ }^{8}$, almost all the loss in sensitivity is recovered by improved noise over expectation. A related observation to the sensitivity decrease is that of charge spreading in Channel 3 - for example, seen in cosmic ray events ${ }^{11}$. We now believe that there are several explanations leading to observed discrepancy in Channel 3 sensitivity. First, Ames has re-examined expectations of the Si:As quantum efficiency (QE) for the actual Channel 3 band pass (Ames tested through a fixed 6.4 $\mu \mathrm{m}$ filter), and conclude that the QE quoted prior to flight was too large, and second, at least in part, the throughput anomaly may be linked to another phenomenon known as "banding" which had been observed but not explained preflight. In addition, two related effects ${ }^{10}$, namely "column pull-down" by a bright source, and a different DC level for a frame containing an extremely bright source (we call this "full array pull-up"), are also probably related to "banding".

In careful measurements of charge collected outside the spot diameter (in an attempt to look at the Channel 4 sensitivity anomaly), Ames could account for only about a 7\% dispersed-charge effect, well less than observed in flight. They forwarded two of the parts to UR for subsequent testing in their test system.

\subsubsection{Quantum Efficiency of Si:As in the $5.8 \mu \mathrm{m}$ Bandpass}

Once the throughput anomaly in flight referred to above was realized, Ames revisited the Si:As literature and generated a new prediction of the quantum efficiency $(\mathrm{QE})$, based on the final choice of detector geometry, doping, etc. as well as the appropriate final wavelength coverage chosen for IRAC. Channel 3 is being operated at wavelengths well below the optimum wavelength of operation for Si:As, namely $7.0 \mu \mathrm{m}$.

RQE (responsive quantum efficiency) predictions were generated using the latest experimental data on the Si:As absorption cross-section ${ }^{12}$. The revised prediction is shown in Figure 3. It shows a dramatically reduced effective QE at the $5.06 \mu \mathrm{m}$ lower edge of the Channel 3 band pass, and an estimated value of about $37 \%$ at the $5.8 \mu \mathrm{m}$ Channel 3 
band center. The detective quantum efficiency - DQE - will always be less than the RQE. Overall, this recalculation is in much better agreement with the sensitivities seen in flight.

Tests of the operating point have been conducted, both on the Channel 3 and 4 flight arrays during IOC, and in the lab at Ames on sister parts, and there is no smoking gun there.

Ames' pre-flight testing was done with flood-illumination optics, which were unable to stimulate the charge spreading or banding effects seen in flight. They also operated with a clocking system which was slower than those used at UR and in the flight instrument. UR repeated some of these tests on the same parts, and these tests are discussed below.

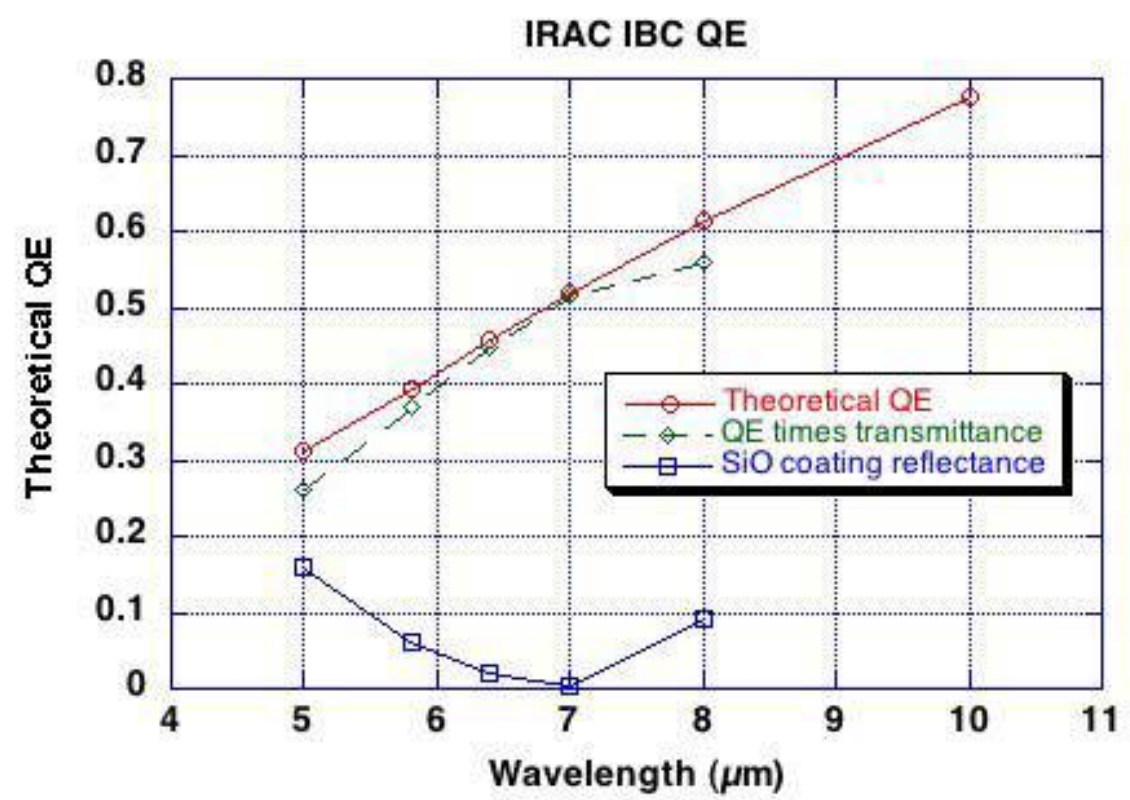

Figure 3. Theoretical Responsive Quantum Efficiency (RQE) vs. Wavelength, for IRAC Channel 3 Arrays

After testing of the flight array candidates at Ames pre-flight, they were returned to Raytheon (formerly SBRC) for cleaning, and packaging onto the GSFC-supplied carriers. Given this sequence, it is conceivable that the cleaning/etching step at SBRC might have altered the array.

\subsubsection{Banding}

Both Channel 3 and 4 show a banding structure around moderate to bright sources. The banding is an apparent extension of the object in four directions, along the rows or the columns. Banding is seen in both stars (point sources) and bright nebulous regions (extended sources). See for example Figure 4, left, where banding in the S140 star formation region is shown at $5.8 \mu \mathrm{m}$. Various theories were proposed for the source of banding, from optical effects both external to the detector and within the detector to electronic cross-talk effects. One such theory is that the banding is due to light scattering inside the detector and inside the epoxy between the detector and multiplexer before being scattered again back into the detector where it is finally absorbed. Tests conducted at UR provide evidence in support of the theory that banding is an optical effect where light is propagating through the detector and epoxy. These tests and results are described briefly below. 
A pin light illuminated a sister array to Channel 3 at wavelengths from 1.25 to $4.5 \mu \mathrm{m}$ with flux levels exceeding $210^{5}$ $\mathrm{DN} / \mathrm{s} /$ pixel, producing a spot roughly 40 pixels in diameter, at an angle of incidence of $\sim 4.4^{\circ}$. A dark mask next to the array blocks out external light in an area adjacent to that containing the source image. See Figure 4 right for a $1.25 \mu \mathrm{m}$ image of banding produced in the lab, with contours overlaid, for comparison with the in-flight image of S140.

For wavelengths from $4.5-10 \mu \mathrm{m}$, a mask with a pinhole was placed in contact with the detector surface, and viewing an external blackbody through cold filters, the spot size was 14-16 pixels in diameter. The ratio of total signal in the band structure to that in the source exhibits a broad peak at wavelengths out to $2.3 \mu \mathrm{m}$ of magnitude $20-25 \%$, declining to $<10 \%$ at 4.2 and $4.5 \mu \mathrm{m}$. There are broad peaks in the ratio which includes at the $20 \%$ level all of the Channel 3 band pass, and another broad peak which includes $50 \%$ of the Channel 4 band pass, although at the $10 \%$ level. These data are presented in Figure 5.

By employing a dark mask, it is clear that light does indeed scatter to all pixels in the array, and not just into the bands. The amount of light scattered into all pixels (excluding the source) at $\lambda=5.6 \mu \mathrm{m}$, for example, is $130 \pm 10 \%$ of the flux scattered into the bands (for example, if $23 \%$ of the source flux is scattered in the bands, then $30 \%$ of the source flux would be scattered to all other pixels). The large amount of scattered light can account for what we call "full array pullup" to the array DC level surrounding a bright source.

There are several sources of uncertainty in these measurements. Non-linearity corrections $(<20 \%)$ have not been applied; the bright source saturated beyond $6.4 \mu \mathrm{m}$, so that a blackbody extrapolation from shorter wavelength data out to $10 \mu \mathrm{m}$ was used; and a constant RQE was assumed (the Channel $4 \mathrm{QE}$ is likely $1.5 \mathrm{x}$ the Channel $3 \mathrm{QE}$, see Figure 3, leading to a ratio of band to source fluxes that is that factor lower).

Figure 4. Left. Banding in Channel 3 image of S140, a protostar embedded in nebulosity. Right: Banding at $1.25 \mu \mathrm{m}$ for a Channel 3 type array. Image is negative (darker is brighter). The source itself is saturated in the pedestal frame, and since a \{source - pedestal\} image is displayed, appears to have 0 signal.
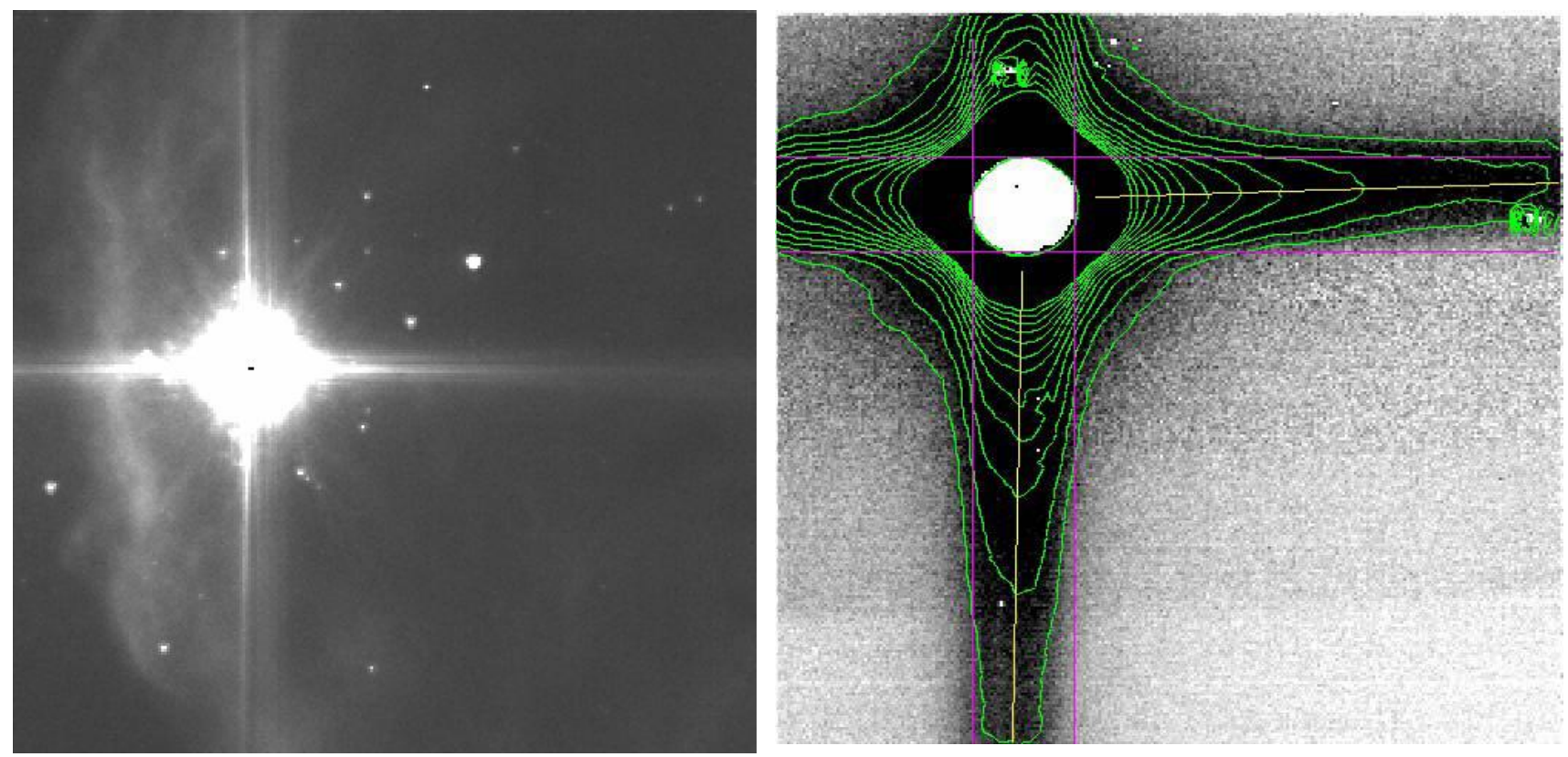
There is no discontinuity in a band that passes under a mask in the short wave lab measurements, nor does the full coverage mask in the long wave lab measurements preclude the existence of a band. UR lab and dewar optics have very little in common with the Spitzer Space Telescope's optics. These points demonstrate that banding is independent of the external optics. The wavelength dependence of banding shown in Figure 5 indicates that it is not an electrical effect. These considerations, taken together, lead us to conclude that the banding is due to light scattering within the detector and more likely between the detector and the multiplexer.

The fact that the epoxy filling that void between the multiplexer and detector array does not have measurable transmission at room temperature (Raytheon, private communication) suggests that the epoxy's transmission at $6 \mathrm{~K}$, the temperature at which these Si:As arrays operate, is quite different. This hypothesis has not been confirmed in the lab.

Full array pull-up (the enhanced signal level over the rest of the array in the presence of a bright source, described above) cannot be due to electronic or multiplexer effects because the flux level varies with pixel distance from the source. In addition, the signals at every pixel (both inside and outside the bands) obey Poisson statistics, typical of photon noise. As noted above, the ratio of the total flux scattered to all the pixels outside of the bands to that in the bands is independent of wavelength. Thus we conclude that full array pull-up or droop is due to light scattering from the source to all the pixels on the array.

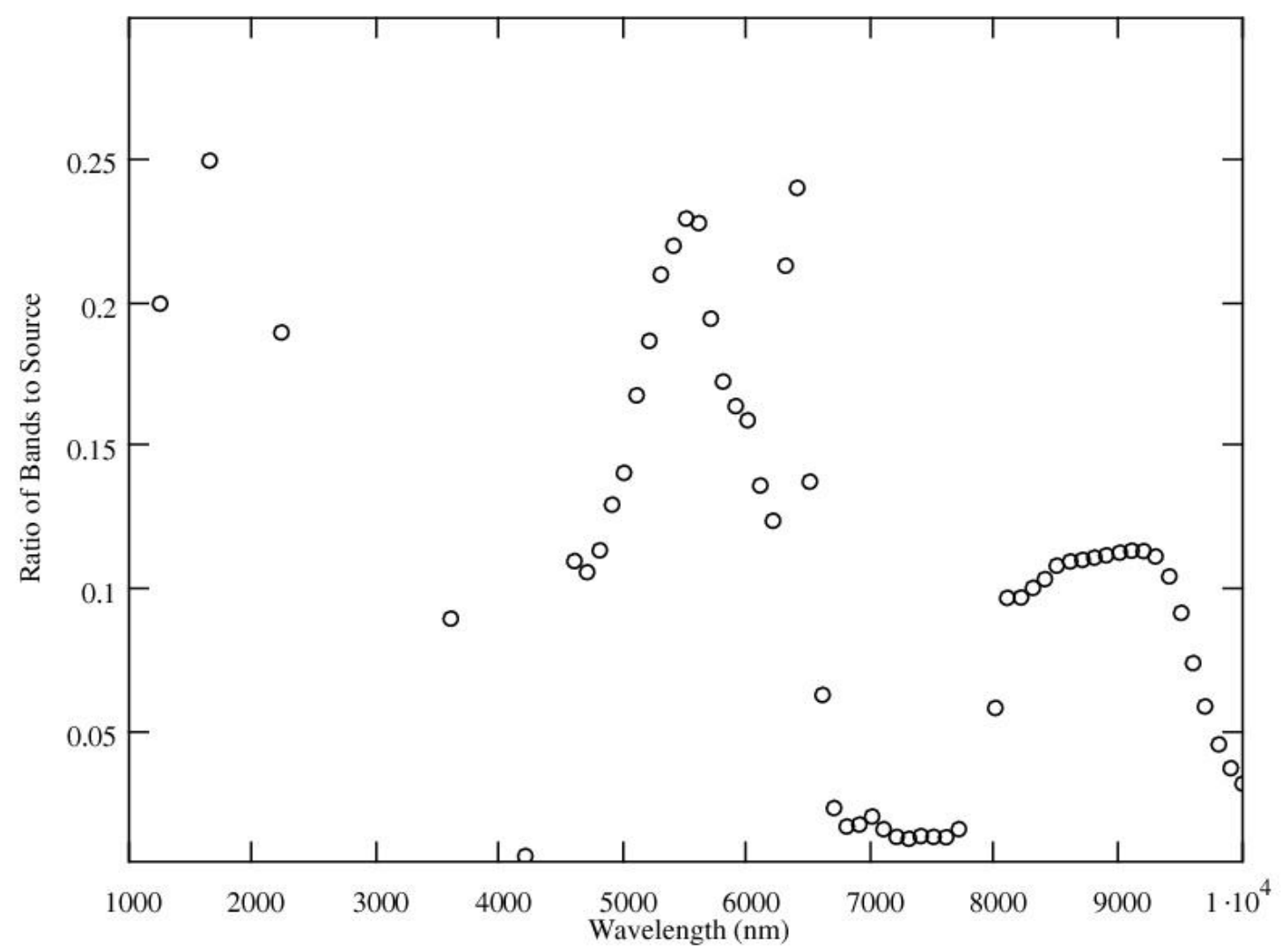

Figure 5. Ratio of \{total signal level in bands to that in source $\}$ as a function of wavelength. Note calibration caveats in text. 


\subsection{Latent Images in the Detector Material}

Prior to flight, substantial effort was directed toward characterization of normal residual (latent) images in the IRAC detector arrays: Benson et al. ${ }^{2}$ showed that they originated in the detector material in the neighborhood of the depletion region for the Channel 1 and 2 arrays, and a variety of tests, both in pre-flight and during IOC were obtained to characterize these as a function of both flux and fluence, for all observing scenarios. During IOC, a globular cluster, 47 Tuc as well as two extremely bright stars were observed to calibrate latent images as a function of stellar magnitude so the Spitzer Science Center team could develop predictive tools to remove or tag these images. These objects covered the range of source brightness that would lead to "normal" latent images in all channels.

\subsection{Optical and Electronic Crosstalk; Multiplexer (Mux) Bleed}

As noted prior to flight ${ }^{1}$, there are several types of crosstalk including optical, electronic, and mux bleed. Optical crosstalk is wholly the result of diffusion in the Channel 1 and 2 detector arrays. Photons are absorbed within a few microns of the light-incident back surface, and diffuse toward the diode for collection. Adjacent pixels can gather some of the charge. Electronic cross-talk is detected every four pixels (every fourth pixels uses the same output amplifier) and is due to finite bandwidth response of the multiplexer: this affects all channels. Mux bleed in the InSb arrays was also well-characterized prior to flight, and occurs along rows as a glow falling off every four columns in a slow exponential trail from a bright star. As discussed by Carey et al. ${ }^{10}$, pipeline corrections for these effects are not $100 \%$ perfect. High dynamic range (HDR) mode observations help in determination of the pixel level in the absence of these anomalies.

\section{SUMMARY}

The spectacular images obtained by Spitzer IRAC (see http://www.spitzer.caltech.edu) are testimony to the unprecedented sensitivity of the four IRAC arrays as well as to the efforts of the Spitzer teams to characterize these arrays completely. Despite several anomalous behaviors, methods of working around the issues have been found, correction algorithms have been developed, and calibration and observational strategies are in place to avoid compromising otherwise spectacular results.

\section{ACKNOWLEDGEMENTS}

This work is based on observations made with the Spitzer Space Telescope, which is operated by the Jet Propulsion Laboratory, California Institute of technology under NASA contract 1407, and by experimental support work at NASA Ames under "Infrared Detector Development Support", FY 04 RTOP 188-02-01 and at the University of Rochester under grant SVS 660011 from Smithsonian Astrophysical Observatory (SAO), which in turn is supported by NASA

through Contract Number 960541 issued by JPL for IRAC. Support for the writing of this paper is under SAO grant SV4-74011.

\section{REFERENCES}

1. Pipher, J.L. et al. 2000, "InSb Arrays for IRAC (Infrared Array Camera) on SIRTF (Space Infrared Telescope Facility)" in Infrared Spaceborne Remote Sensing VIII, eds. M. Stojnik \& B.F. Andresen, Proc. SPIE, 4131, 218.

2. Benson, R.G. et al, 2000, "Spatial distribution of hole traps and image latency in InSb focal plane arrays" in Infrared Spaceborne Remote Sensing VIII, eds. M. Stojnik \& B.F. Andresen, Proc. SPIE, 4131, 171.

3. Hora, J.L. et al. 2000, "Calibration and Performance of the Infrared Array Camera (IRAC)" Proc. SPIE, 4131, $13-25$.

4. Hora , J.L.et al. 2001, "Calibration of the Infrared Array Camera (IRAC) for SIRTF" in Proceedings of the Conference The Calibration Legacy of the ISO Mission, VI LSPA, Spain, ESA SP-481, 73. 
5. Hora, J.L. et al. 2003, "Performance of the Infrared Array Camera for SIRTF during Integration and Test" Infrared Space Telescopes and Instruments, SPIE Proc. 4850, 83.

6. McMurray, R.E. Jr. et al. 2000, "Si:As IBC Array Performance for SIRTF/IRAC" in Infrared Spaceborne Remote Sensing VIII, eds. M. Stojnik \& B.F. Andresen, Proc. SPIE, 4131, 62.

7. Fazio, G.G. et al. 2004, "In-flight Performance of the Infrared Arrays Camera on the Spitzer Space Telescope", Astrophys. J. Suppl. Special Spitzer issue.

8. Hora, J.L. et al. 2004, "In-flight Performance and Calibration of the Infrared Arrays Camera (IRAC) for SIRTF - Spitzer Space Telescope", this volume.

9. Hoffman, A.W. et al. 1998, "Near IR Arrays for Ground-based and Space-based Astronomy" in Infrared Astronomical Instrumentation, ed. A. Fowler, Proc. SPIE 3354, 24-29.

10. Carey, S.J. et al., 2004, "Observing with the Infrared Array Camera (IRAC) on the Spitzer Space Telescope", this volume.

11. Patten, B.M. et al., 2004, "Normal and Unusual Transient Events in IRAC Images", this volume.

12. Geist, J. 1989, "Infrared absorption cross-section of arsenic in silicon in the impurity band region of concentration," Appl. Opt. 28, 1193. 